\title{
Evaluation of Jagged2 and Gli1 expression and their correlation with prognosis in human hepatocellular carcinoma
}

\author{
JUNLI ZHANG* ${ }^{*}$, KANGSHENG TU* , WEI YANG, CHAO LI, YINGMIN YAO, XIN ZHENG and QINGGUANG LIU \\ Department of Hepatobiliary Surgery, The First Affiliated Hospital of Xi'an Jiaotong University, \\ Xi'an, Shaanxi 710061, P.R. China
}

Received September 22, 2013; Accepted April 16, 2014

DOI: $10.3892 / \mathrm{mmr} .2014 .2246$

\begin{abstract}
Jagged2 is closely associated with numerous congenital diseases and has an important role in multiple malignancies. It has been identified that Jagged 2 is a sonic hedgehog-regulated factor. However, its expression and correlation with Gli1 in hepatocellular carcinoma (HCC) remain unknown. A total of 58 samples of surgically resected paired HCC and normal tumor-adjacent tissues were collected, and the Jagged 2 and Gli1 expression was detected in the samples using immunohistochemical staining. The correlation between Jagged 2 and Gli1 protein expression, and their correlation with the clinicopathological features of HCC were analyzed. The protein expression of Jagged2 and Gli1 were significantly upregulated in HCC tissues compared with the normal tumor-adjacent tissues $(\mathrm{P}<0.001$, respectively), and Jagged 2 expression was positively correlated with Glil protein in HCC tissues $(\mathrm{r}=0.643, \mathrm{P}<0.001)$. Jagged 2 and Gli1 protein were expressed at significantly higher levels in patients with intrahepatic metastasis, high histological grade and advanced tumor-node-metastasis stage $(\mathrm{TNM})$ stage $(\mathrm{P}<0.05$, respectively). With the Cox proportional hazard regression mode, the independent factors predictive of poor long-term HCC survival following radical liver resection included high expression of Jagged2, advanced TNM stage and high histological grade $(\mathrm{P}<0.05)$. In HCC, high expression of Jagged 2 was closely correlated with poor clinicopathological features, and it may therefore be a potential prognosis predictor for patients with HCC.
\end{abstract}

Correspondence to: Professor Qingguang Liu or Dr Xin Zheng, Department of Hepatobiliary Surgery, The First Affiliated Hospital of Xi'an Jiaotong University, Xi'an, Shaanxi 710061, P.R. China

E-mail: liuqingguang@vip.sina.com

E-mail: xin.zheng.xjtu@gmail.com

*Contributed equally

Key words: Jagged2, Notch signaling, Gli1, Hedgehog signaling, hepatocellular carcinoma

\section{Introduction}

Hepatocellular carcinoma (HCC) is the third leading cause of cancer-related mortality worldwide, with up to 75,0000 novel cases reported annually (1). Despite the fact that the major risk factors, including viral hepatitis (B and C) and/or alcohol abuse, have been identified, the therapeutic options remain limited due to incomplete understanding of the cellular and molecular mechanisms involved in the pathogenesis of HCC (2). Therefore, further investigation of the mechanisms involved in the genesis and development of HCC is important for identifying novel therapeutic approaches to improve the clinical outcomes for patients with HCC.

Notch signaling is a classical signaling pathway, which has an important role in regulating cell fate decisions, proliferation and apoptosis of multiple cell lineages (3). Notch signaling comprises several transmembrane receptors and their ligands, transcription factors, as well as negative and positive modifiers. Notch receptors (Notch 1-4) and two groups of ligands, Jagged (Jagged1 and Jagged2) and Delta-like (DLL1, DLL3, DLL4), have been identified in mammals (4). Notch signaling has been identified to be associated with numerous types of tumor, including human HCC, as evidenced by its contribution to the formation of liver tumors in mice (5). One Notch signaling ligands, Jagged2, is expressed widely in human organs, including the heart, skeletal muscle and pancreas (6). It has been demonstrated that a missense mutation in the Jagged 2 gene leads to mouse syndactylism with digit malformation (7). Mice with null mutations of Jagged 2 also exhibit a number of defects, including cleft palate, syndactyly and thymic abnormalities (6). Additionally, Jagged2 also appears to have an important role in various diseases, including malignant tumors. Jagged2 was reported to mediate lung adenocarcinoma epithelial-mesenchymal transition (EMT) and metastasis in mice (8). In hypoxic conditions, Jagged2 promoted breast cancer metastasis and self-renewal of cancer stem-like cells (9). Notch3 and Jagged2 contribute to gastric cancer development and glandular differentiation (10). Notably, the overexpression of Jagged 2 has been identified in $>90 \%$ of pancreatic cancer cell lines (11). However, the role of Jagged2 in the genesis and development of HCC remains elusive.

Sonic hedgehog (Shh) signaling has a key role in the regulation of embryogenesis, adult tissue homeostasis and carcinogenesis $(12,13)$. It is activated by Shh ligand binding 
to the Patched-1 receptor, which relieves the repression of transducer protein Smoothened and subsequently triggers activation of the Gli family of transcription factors, including Gli1. The activated Hedgehog signaling regulates cell proliferation, survival, angiogenesis and EMT in HCC (14). A transcriptome analysis identified Jagged 2 as a sonic hedgehog-regulated factor, where inhibition of Shh signaling by a dominant-negative version of Gli3 (Gli3 ${ }^{\text {Rep }}$ ) resulted in the loss of Jagged 2 expression. Conversely, constitutive activation of the Shh pathway, using Gli3 ${ }^{\text {Act }}$ resulted in the upregulation of Jagged2 $(13,15)$. Nevertheless, the correlation between Gli1 and Jagged2 in human HCC remains poorly understood.

In the present study, the Jagged2 expression and its relation with Gli1 protein in human HCC was investigated, and then the correlation between Jagged2 expression and the clinicopathological features of HCC were analyzed, detailing its potential as a prognostic marker for HCC patients.

\section{Materials and methods}

Patients and tissue specimens. A total of 58 patients were recruited, including 15 females and 43 males (mean age, 51.7 years; range, 24-78). The patients had not been treated with preoperative chemotherapy or interventional embolization. All of the patients received curative liver resection in the Department of Hepatobiliary Surgery, First Affiliated Hospital of Medical College of Xi'an Jiaotong University, (Xi'an, China) between December 2009 and June 2010. All of the procedures were approved by the Xi'an Jiaotong University Ethics Committee and informed consent forms were signed by each patient. A total of $58 \mathrm{HCC}$ tissues and matched normal tumor-adjacent tissues $(>2 \mathrm{~cm}$ distance from the margin of the resection) were collected with an area of $0.5 \times 0.5 \mathrm{~cm}$ and stored immediately in paraformaldehyde for immunohistochemistry. All of the patients were followed-up, either via visits or telephone contact, with a median follow-up time of 23 months (range, 3-36 months) after liver resection. The data of clinical features was obtained from the medical records of each patient. The maximum diameter of the tumor, intrahepatic metastasis, histological Edmonson classification and tumor-node-metastasis (TNM) stage were gathered from the pathological records and confirmed by two experienced pathologists who were blinded to the clinical data and the results of immunohistochemical staining.

Immunohistochemical analysis. Horseradish peroxidase staining was applied for immunohistochemical analysis. All of the paraformaldehyde-fixed paraffin sections were incubated at $60^{\circ} \mathrm{C}$ for $\geq 4 \mathrm{~h}$. Next, the sections were dewaxed in dimethylbenzene and rehydrated in alcohol of diminishing concentrations. All of the sections were then placed into citrate buffer and boiled for 15 min for antigen retrieval. A total of $3.0 \%$ hydrogen peroxide was utilized for eliminating the endogenous peroxidase. The sections were then blocked by $10 \%$ goat serum at $37^{\circ} \mathrm{C}$ for $30 \mathrm{~min}$ and incubated at $4{ }^{\circ} \mathrm{C}$ overnight with primary antibody directed against Jagged2 (1:100; Santa Cruz Biotechnology Inc., Santa Cruz, CA, USA; sc-5604) or Gli1 (1:100; Santa Cruz Biotechnology Inc.; sc-20687). The biotinylated goat anti-rabbit secondary antibody (Beijing
A

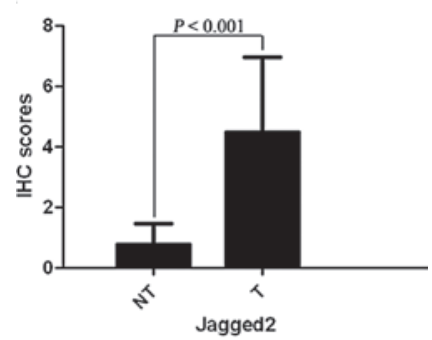

B

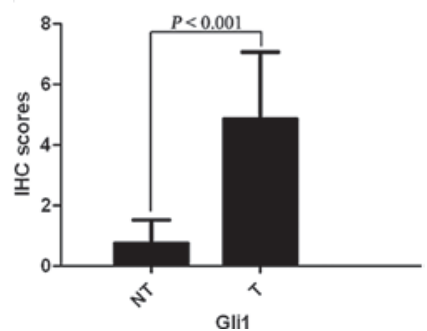

Figure 1. Expression level of Jagged 2 and Gli1 in T and NT tissues Expression level of (A) Jagged 2 and (B) Gli1 in HCC tissues was significantly higher than those in normal NT tissues. HCC, hepatocellular carcinoma; T, HCC tissues; NT, normal tumor-adjacent tissues.

Zhongshan Goldenbridge Biotechnology Co., Ltd., Beijing, China) was used to detect the primary antibody at $37^{\circ} \mathrm{C}$ for $45 \mathrm{~min}$. Next, the horseradish peroxidase-streptavidin conjugate was used to react with biotinylated secondary antibody at $37^{\circ} \mathrm{C}$ for $30 \mathrm{~min}$. The sections were reacted with diaminobenzidine and counterstained with hematoxylin. Finally, the sections were dehydrated in increasing concentrations of alcohol, transparentized by dimethylbenzene and coverslipped onto glass slides.

All of the stained sections were observed by two independent experienced pathologists in a blinded manner. Each stained section obtained a final score based on the intensity and percentage of positive cells following semi-quantitative assessment. The staining intensity was grouped into four grades: 0 , negative staining; 1 , weakly positive staining; 2 , moderately positive staining; and 3 , strongly positive staining. The stained sections with different percentages of positive cells were scored appropriately: 0 (<5\%), $1(5-25 \%), 2(26-50 \%), 3$ $(51-75 \%)$ and $4(>75 \%)$. These two scores were the mean of ten different high magnification (x400) fields and were multiplied to calculate the final score of each stained section. The sections with a total score of $>1$ were defined as exhibiting positive staining for the above proteins (16).

Statistical analysis. Each quantitative value was expressed as the mean \pm standard deviation or the median. Comparison of Jagged 2 or Gli1 protein expression between HCC tissue and normal tumor-adjacent tissue was calculated by a paired-samples t-test. Spearman's rank correlation coefficient test was used to analyze the correlation between Jagged 2 and Gli1 protein expression. The differences in Jagged 2 or Gli1 expression in the HCC tissues with different clinical features were compared by the Mann-Whitney U-test. The Kaplan-Meier method was used to obtain the survival curves of different clinical characteristics and the differences between these survival curves were analyzed 
Table I. Correlation between the clinicopathological characteristics and protein expression of Jagged 2 and Gli1 in the 58 hepatocellular carcinoma patients.

\begin{tabular}{lcccc}
\hline Characteristic & U-value (Jagged2) & P-value (Jagged2) & U-value (Gli1) & P-value (Gli1) \\
\hline Age $(<50 / \geq 50)$ & 341.0 & 0.214 & 393.0 & 0.679 \\
Sex (male/female) & 306.5 & 0.770 & 302.0 & 0.705 \\
AFP $(<400 / \geq 400$ ug/dl) & 222.0 & 0.457 & 194.0 & 0.183 \\
HBsAg (positive/negative) & 247.5 & 0.389 & 256.0 & 0.479 \\
Cirrhosis (yes/no) & 246.0 & 0.554 & 382.5 & 0.749 \\
Tumor size (<5/ $\geq 5$ cm) & 350.5 & 0.263 & 149.5 & 0.583 \\
Intrahepatic metastasis (yes/no) & 126.0 & 0.007 & 273.5 & 0.024 \\
Hepatic capsule invasion (yes/no) & 298.5 & 0.503 & 177.0 & 0.255 \\
Portal vein tumor thrombus (yes/no) & 185.5 & 0.748 & 230.0 & 0.618 \\
TNM stage (I-II/III-IV) & 220.5 & 0.001 & 256.5 & 0.007 \\
Edmonson classification (I-II/III-IV) & 209.0 & 0.004 & 0.034
\end{tabular}

AFP, $\alpha$-fetoprotein.

Table II. Univariate analysis of Kaplan-Meier method.

\begin{tabular}{lrr}
\hline Variable & $\chi^{2}$-value & P-value \\
\hline Jagged2 expression (low/high) & 17.728 & 0.000 \\
Gli1 expression (low/high) & 4.612 & 0.032 \\
AFP $(<400 / \geq 400 \mathrm{ug} / \mathrm{dl})$ & 4.585 & 0.032 \\
Tumor size $(<5 / \geq 5 \mathrm{~cm})$ & 4.491 & 0.034 \\
Intrahepatic metastasis (yes/no) & 13.837 & 0.000 \\
TNM stage (I-II/III-IV) & 20.419 & 0.000 \\
Edmonson classification (I-II/III-IV) & 12.014 & 0.001 \\
\hline
\end{tabular}

AFP, $\alpha$-fetoprotein; TNM, tumor-node-metastasis.

Table III. Cox proportional hazard regression mode.

\begin{tabular}{|c|c|c|c|c|c|c|}
\hline Variable & $\mathrm{B}$ & SE & Wald & $\operatorname{Exp}(B)$ & P-value & $95 \% \mathrm{CI}$ \\
\hline Gli1 expression (low/high) & -0.711 & 0.428 & 2.755 & 0.491 & 0.097 & $0.212-1.137$ \\
\hline Jagged 2 expression (low/high) & 1.309 & 0.417 & 9.866 & 3.701 & 0.002 & $1.636-8.375$ \\
\hline $\operatorname{AFP}(<400 / \geq 400 \mathrm{ug} / \mathrm{dl})$ & 0.459 & 0.452 & 1.030 & 1.582 & 0.310 & $0.653-3.834$ \\
\hline Tumor size $(<5 / \geq 5 \mathrm{~cm})$ & -0.329 & 0.455 & 0.525 & 0.719 & 0.469 & $0.295-1.753$ \\
\hline Intrahepatic metastasis (yes/no) & 0.873 & 0.460 & 3.593 & 3.749 & 0.058 & $0.971-5.901$ \\
\hline TNM stage (I-II/III-IV) & 1.979 & 0.582 & 11.567 & 7.234 & 0.001 & $2.313-22.626$ \\
\hline Edmonson classification (I-II/III-IV) & 1.321 & 0.411 & 10.352 & 2.393 & 0.001 & $1.676-8.385$ \\
\hline
\end{tabular}

B, partial regression coefficient; SE, partial regression coefficient standard error; AFP, $\alpha$-fetoprotein; TNM, tumor-node-metastasis; CI, confidence interval.

using a log-rank test. The Cox proportional hazard regression model was applied to determine the independent prognostic factors in multivariate survival analysis. SPSS 17.0 software (SPSS, Inc., Chicago, IL, USA) was used in this statistical analysis and $\mathrm{P}<0.05$ was considered to indicate a statistically significant difference.

\section{Results}

Expression of Jagged 2 and Glil in HCC and matched normal tumor-adjacent tissues. Immunohistochemical staining demonstrated that Jagged 2 protein expression in HCC tissues was found to be significantly higher than that in the matched 


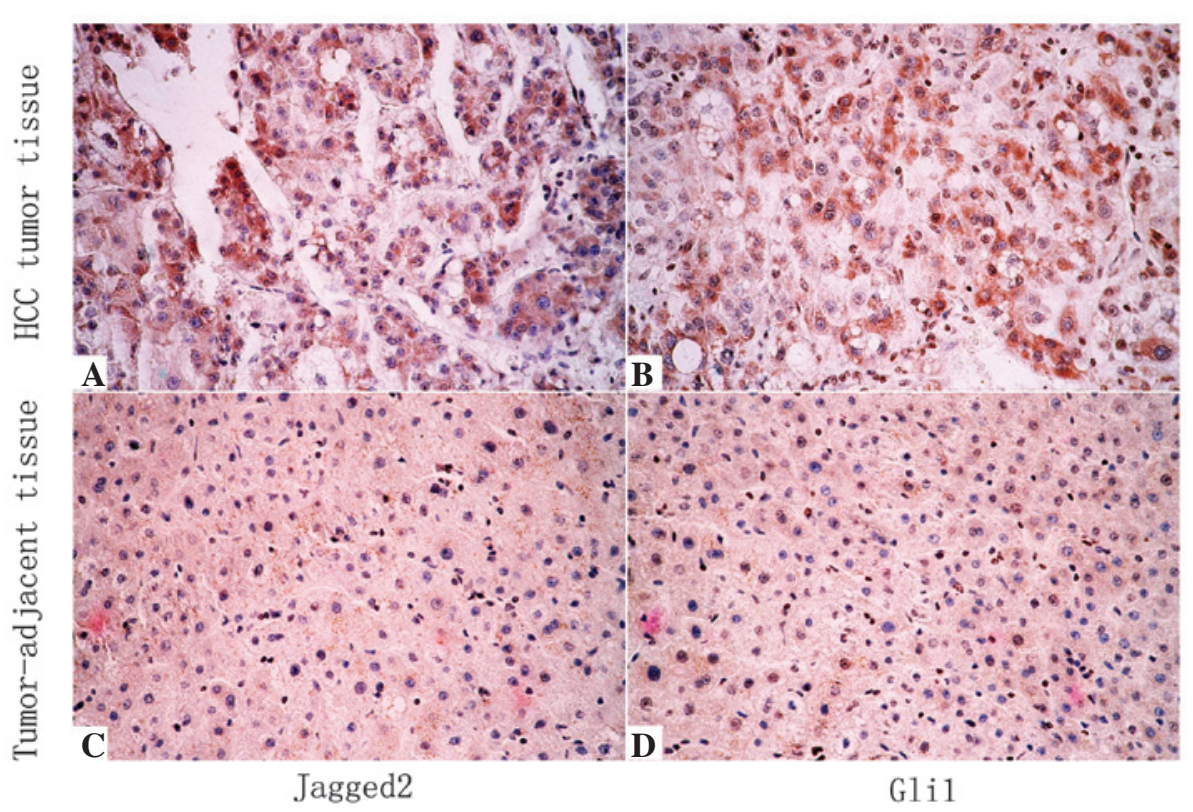

Figure 2. Immunohistochemical staining of Jagged2 and Gli1 in HCC tissues and normal tumor-adjacent tissues. In the cases of high Jagged2 protein expression (A), there was strong staining of Gli1 in the same HCC tissue section (B). By contrast, in the case of low Jagged2 protein expression (C), there was no detectable Gli1 protein expression in the same normal tumor-adjacent tissue section (D) (original magnification, x400; staining, streptavidin peroxidase). HCC, hepatocellular carcinoma.

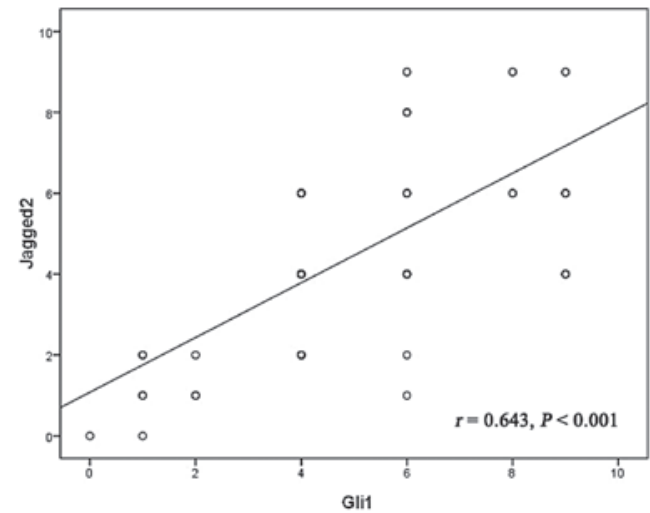

Figure 3. In the Spearman's rank correlation coefficient test, Jagged2 demonstrated a positive correlation with Gli1 protein expression in hepatocellular carcinoma tissues $(\mathrm{r}=0.643, \mathrm{P}<0.001)$.

normal tumor-adjacent tissues $(4.47 \pm 2.48$ vs. $0.78 \pm 0.68$; $\mathrm{P}<0.001$; Fig. 1A). The same was observed for Gli1 protein expression $(4.84 \pm 2.24$ vs. $0.74 \pm 0.79 ; \mathrm{P}<0.001$; Fig. 1B). The positive staining of Jagged 2 was observed mainly in the cytoplasm, while Gli1 was localized in the cytoplasm and nuclei in HCC cells (Fig. 2A-B).

Correlation between Jagged 2 and Gli1. A previous study indicated that there is a correlation between Jagged2 and Gli1 expression in various malignancies; however, this association has not been observed in HCC (15). Using the Spearman's rank correlation coefficient test, Jagged2 was demonstrated to be positively correlated with Gli1 protein expression ( $\mathrm{r}=0.643$, $\mathrm{P}<0.001$; Fig. 3)

Correlation between Jagged2 or Glil protein expression in HCC tissues and clinicopathological features. The correlation
A

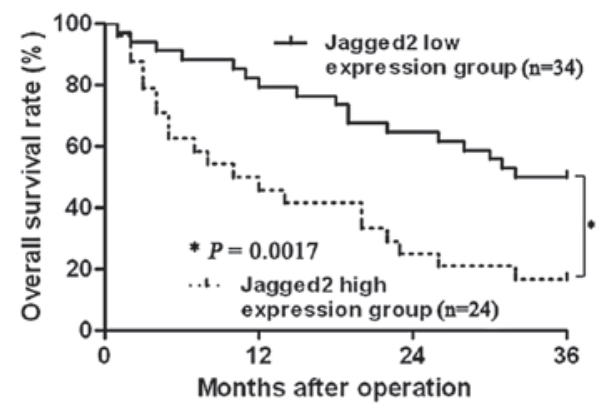

B

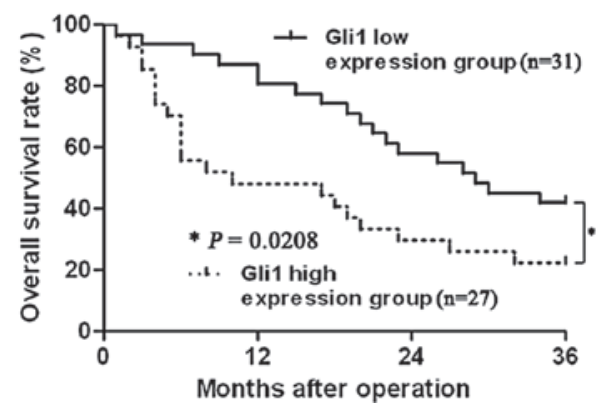

Figure 4. Kaplan-Meier overall survival curves of patients with hepatocellular carcinoma according to the level of Jagged 2 and Gli1 protein. (A) The Jagged 2 high expression group $(n=24)$, Jagged 2 expression level $\geq$ median value of Jagged 2 expression level; low Jagged 2 expression group $(n=34)$, Jagged 2 expression level < median value of Jagged 2 expression level. (B) The high Gli1 expression group $(n=27)$, Glil expression level $\geq$ median value of Gli1 expression level; low Gli1 expression group ( $n=31)$, Gli1 expression level $<$ median value of Glil expression level.

between Jagged 2 or Gli1 protein expression and clinicopathological parameters in $58 \mathrm{HCC}$ patients was analyzed by the Mann-Whitney U-test, and the results are shown in Table I. Jagged 2 protein expression was significantly correlated with intrahepatic metastasis $(\mathrm{P}=0.007)$, advanced TNM stage $(\mathrm{P}=0.001)$ and high Edmonson pathological classification 
$(\mathrm{P}=0.004)$. Gli1 protein expression was also closely associated with intrahepatic metastasis $(\mathrm{P}=0.024)$, advanced TNM stage $(\mathrm{P}=0.007)$ and high Edmonson pathological classification $(\mathrm{P}=0.034)$.

Analysis of risk factors for patient survival. The Kaplan-Meier method was used in univariate analysis. Median values of Jagged 2 and Gli1 were used as cut-off points to divide the patients into two groups: The low expression group and the high expression group. As demonstrated in Table II, Jagged2, Gli1, $\alpha$-fetoprotein (AFP level), maximum tumor diameter, intrahepatic metastasis, TNM stage and Edmonson pathological classification were associated with patient 3-year overall survival $(\mathrm{P}<0.05$, respectively). The survival curves of variables are demonstrated in Fig. 4. With further investigation, Cox proportional hazard regression analysis was conducted with the seven variables that were previously determined, to select the potential risk factors for determining prognosis. The results demonstrated that TNM stage, Edmonson pathological classification and Jagged 2 expression were potential risk factors, which affected the prognosis of HCC patients $(\mathrm{P}<0.05$, respectively; Table III).

\section{Discussion}

As one of the ligands of Notch signaling, Jagged 2 expression was found to be associated with a variety of tumor types. However, there is a controversy regarding the effect of Jagged2 on tumorigenesis and tumor development. One study reported that Jagged2 regulated the expression of cytokines, which promote antitumor immunity (17). However, another study rejected this and demonstrated that Jagged2 enhances cell growth, invasion and migration in two uveal melanoma cell lines (Mel285 and Mel290) (18). In the cutaneous melanoma cell line, Jagged 2 was the most markedly overexpressed gene in the highly invasive clone, with an RNA level 15 -fold higher than that in the less invasive cells (19). Furthermore, when Jagged 2 was induced at the transcriptional level under hypoxic conditions, it was significantly correlated with angiogenic processes in breast cancer, renal cell carcinoma and epithelial tumor cells (20). However, to the best of our knowledge no study has been conducted regarding the association between HCC and Jagged2. In the present study, the results identified that Jagged 2 expression in HCC tissues was notably higher than that in the matched normal tumor-adjacent tissues. In addition, the clinical pathological parameter analysis demonstrated that Jagged2 was significantly correlated with intrahepatic metastasis, advanced TNM stage and high Edmonson pathological classification, suggesting that Jagged 2 may have an important role in tumorgenesis and tumor development. This observation is consistent with those of previous studies concerning the oncogenic effect of Jagged 2 in several malignant tumor types (8-11). It was also identified that patients with low Jagged 2 expression had an improved outcome compared with patients with high Jagged2 expression. Furthermore, Cox proportional hazard regression mode analysis suggested that Jagged 2 was an independent factor for the prediction of poor long-term HCC survival following radical liver resection.

Hedgehog/Glil signaling pathway is involved in a variety of human cancer types and aberrant activation of this signaling has also been identified to be associated with HCC (21-23). Recent studies identified that the transcription factor Gli1 was associated with poor prognosis among the patients with $\mathrm{HCC}$ and the expression of the Glil gene in tumor tissues was significantly correlated with disease-free survival and overall survival rates (24). Using immunohistochemical staining, the present study demonstrated that Glil expression in HCC tissues was higher than that in the matched tumor-adjacent tissues. Gli1 was significantly correlated with intrahepatic metastasis, advanced TNM stage and high Edmonson pathological classification, and the 3-year overall survival of the patients with Gli1 high expression was lower than that in those with low Glil expression. Additionally, the AFP level, maximum tumor diameter, intrahepatic metastasis, TNM stage and Edmonson pathological classification were associated with the 3 -year overall survival. According to the clinical research, HCC patients with advanced TNM stage and high Edmonson pathological classification have a poor long-term survival.

Hedgehog signaling induced Jagged 2 upregulation and tumor growth factor- $\beta 1$ secretion to promote the motility and invasiveness of cancer cells (18). A previous transcriptome analysis identified Jagged 2 as a sonic hedgehog-regulated factor (15). However, the accurate mechanisms involved in Hedgehog signaling-induced Jagged 2 upregulation remain unclear. In the present study, it was demonstrated that Jagged2 protein expression was positively correlated with Gli1. This result indicated that Gli1, the key transcriptional factor in Shh signaling, may regulate Jagged 2 expression in HCC.

In conclusion, the present study demonstrated that Jagged 2 and Gli1 were overexpressed in HCC tissues and Jagged 2 was positively correlated with Gli1 protein expression. High-expression of Jagged2 or Glil was associated with a poor 3-year overall survival in HCC patients and Jagged2 acted as an independent predictor of an unfavorable prognosis. Elucidating the regulation between Gli1 and Jagged2 expression, and the mechanisms involved in promoting HCC invasion and metastasis by Jagged2, requires further investigation.

\section{Acknowledgements}

This study was supported by grants from the National Natural Scientific Foundation of China (grant nos. 81272645 and 81072052 to Qingguang Liu and 81071897 to Yingmin Yao), the Research Fund for the doctoral Program of High Education of China from Ministry of Education (grant no. 20120201120090 to Xin Zheng), the Fundamental Research Funds for the Central Universities sponsored by Xian Jiaotong University to Xin Zheng, the Clinical medicine specialty of seven year clinical research and the Innovation Fund for students granted by the First Affiliated Hospital of Xi'an Jiaotong University (13ZD18).

\section{References}

1. Guo C, Liu Q, Zhang L, Yang X, Song T and Yao Y: Double lethal effects of fusion gene of wild-type p53 and JunB on hepatocellular carcinoma cells. J Huazhong Univ Sci Technolog Med Sci 32: 663-668, 2012.

2. Tu K,Zheng X,ZhouZ, et al: Recombinant human adenovirus-p53 injection induced apoptosis in hepatocellular carcinoma cell lines mediated by p53-Fbxw7 pathway, which controls c-Myc and cyclin E. PLoS One 8: e68574, 2013. 
3. Suwanjunee S, Wongchana W and Palaga T: Inhibition of gamma-secretase affects proliferation of leukemia and hepatoma cell lines through Notch signaling. Anticancer Drugs 19 : 477-486, 2008

4. Gao J, Dong Y, Zhang B, et al: Notch1 activation contributes to tumor cell growth and proliferation in human hepatocellular carcinoma HepG2 and SMMC7721 cells. Int J Oncol 41: 1773-1781, 2012.

5. Villanueva A, Alsinet C, Yanger $\mathrm{K}$, et al: Notch signaling is activated in human hepatocellular carcinoma and induces tumor formation in mice. Gastroenterology 143: 1660-1669, 2012.

6. Deng Y, Madan A, Banta AB, Friedman C, Trask BJ, Hood L and Li L: Characterization, chromosomal localization, and the complete 30-kb DNA sequence of the human Jagged2 (JAG2) gene. Genomics 63: 133-138, 2000.

7. Jiang R, Lan Y, Chapman HD, et al: Defects in limb, craniofacial, and thymic development in Jagged 2 mutant mice. Genes Dev 12: 1046-1057, 1998

8. Yang Y, Ahn YH, Gibbons DL, et al: The Notch ligand Jagged 2 promotes lung adenocarcinoma metastasis through a miR-200-dependent pathway in mice. J Clin Invest 121: $1373-1385,2011$

9. Xing F, Okuda H, Watabe M, et al: Hypoxia-induced Jagged2 promotes breast cancer metastasis and self-renewal of cancer stem-like cells. Oncogene 30: 4075-4086, 2011.

10. Kang H, An HJ, Song JY, Kim TH, Heo JH, Ahn DH and Kim G: Notch3 and Jagged 2 contribute to gastric cancer development and to glandular differentiation associated with MUC2 and MUC5AC expression. Histopathology 61: 576-586, 2012.

11. Mullendore ME, Koorstra JB, Li YM, et al: Ligand-dependent Notch signaling is involved in tumor initiation and tumor maintenance in pancreatic cancer. Clin Cancer Res 15: 2291-2301, 2009.

12. Zheng X, Vittar NB, Gai X, et al: The transcription factor GLI1 mediates TGF $\beta 1$ driven EMT in hepatocellular carcinoma via a SNAI1-dependent mechanism. PLoS One 7: e49581, 2012.
13. Katoh $\mathrm{Y}$ and Katoh $\mathrm{M}$ : Hedgehog signaling pathway and gastrointestinal stem cell signaling network. Int J Mol Med 18: 1019-1023, 2006.

14. Robbins DJ, Fei DL and Riobo NA: The Hedgehog signal transduction network. Sci Signal 5: re6, 2012.

15. Rabadán MA, Cayuso J, Le Dréau G, et al: Jagged2 controls the generation of motor neuron and oligodendrocyte progenitors in the ventral spinal cord. Cell Death Differ 19: 209-219, 2012.

16. Tu K, Zheng X, Zan X, Han S, Yao Y and Liu Q: Evaluation of Fbxw7 expression and its correlation with the expression of c-Myc, cyclin E and p53 in human hepatocellular carcinoma. Hepatol Res 42: 904-910, 2012.

17. Choi K, Ahn YH, Gibbons DL, et al: Distinct biological roles for the Notch ligands Jagged-1 and Jagged-2. J Biol Chem 284: 17766-17774, 2009.

18. Asnaghi L, Handa JT, Merbs SL, Harbour JW and Eberhart CG: A role for Jag2 in promoting uveal melanoma dissemination and growth. Invest Ophthalmol Vis Sci 54: 295-306, 2013.

19. Gütgemann A, Golob M, Müller S, Buettner R and Bosserhoff AK Isolation of invasion associated cDNAs in melanoma. Arch Dermatol Res 293: 283-290, 2001

20. Pietras A, von Stedingk K, Lindgren D, Påhlman S and Axelson H: JAG2 induction in hypoxic tumor cells alters Notch signaling and enhances endothelial cell tube formation. Mol Cancer Res 9: 626-636, 2011.

21. Xie J, Murone M, Luoh SM, et al: Activating Smoothened mutations in sporadic basal-cell carcinoma. Nature 391: 90-92, 1998.

22. Karhadkar SS, Bova GS, Abdallah N, et al: Hedgehog signaling in prostate regeneration, neoplasia and metastasis. Nature 431 707-712, 2004

23. Velcheti $\mathrm{V}$ and Govindan R: Hedgehog signaling pathway and lung cancer. J Thorac Oncol 2: 7-10, 2007.

24. Che L, Yuan YH, Jia J and Ren J: Activation of sonic hedgehog signaling pathway is an independent potential prognosis predictor in human hepatocellular carcinoma patients. Chin J Cancer Res 24: 323-331, 2012. 